\title{
ON THE ANTIPODE OF A CO-FROBENIUS (CO)QUASITRIANGULAR HOPF ALGEBRA
}

\author{
MARGARET BEATTIE AND DANIEL BULACU
}

\begin{abstract}
We extend to the co-Frobenius case a result of Drinfeld and Radford related to the fourth power of the antipode of a finite dimensional (co) quasitriangular Hopf algebra.
\end{abstract}

\section{Introduction and Preliminaries}

Throughout this paper, $H$ will denote a (not necessarily finite dimensional) coFrobenius Hopf algebra over a field $k$. All maps are assumed to be $k$-linear. We use the conventional Sweedler-Heyneman notation for the Hopf algebra comultiplication: $\Delta(h)=h_{1} \otimes h_{2}, h \in H$ (summation understood). As usual, the $H^{*}$-bimodule structure on $H$ and the $H$-bimodule structure on $H^{*}$ are given by

$$
l^{*} \rightarrow h<m^{*}=m^{*}\left(h_{1}\right) h_{2} l^{*}\left(h_{3}\right) \text { and }\left(h \rightarrow m^{*}<l\right)(m)=m^{*}(l m h)
$$

for all $h, l, m \in H, l^{*}, m^{*} \in H^{*}$. The antipode of $H$ is denoted $S$ with composition inverse $S^{-1}$. The group of grouplike elements of $H$ is denoted $G(H)$ and the grouplikes of $H^{0}$, namely the set of algebra maps from $H$ to $k$, by $G\left(H^{0}\right)$. We assume familiarity with the basic theory of Hopf algebras; see [4, 10, 14, for example.

In this short note, we see that for $H$ co-Frobenius and quasitriangular (coquasitriangular), the grouplike elements in $H$ ( $H^{*}$ respectively) which define $S^{4}$ as an inner (co-inner) automorphism can be expressed in terms of the modular elements in $H$ and $H^{*}$, thus extending results of Drinfeld and Radford.

Recall that a Hopf algebra $H$ is co-Frobenius if $H^{* \text { rat }}$, the unique maximal rational submodule of $H^{*}$, is nonzero, or, equivalently, if the space of left or right integrals for $H$, denoted $\int_{l}^{H^{*}}$ and $\int_{r}^{H^{*}}$ respectively, is nonzero. It was shown in [3] that $H$ contains a distinguished grouplike element $a$, which we also call the modular element of $H$, such that for all $\lambda \in \int_{l}^{H^{*}}$,

$$
\lambda\left(h_{1}\right) h_{2}=\lambda(h) a^{-1} \text { and } \lambda \circ S^{2}=a^{-1} \rightarrow \lambda<a .
$$

From the definition of a left integral and from (1.1), we have that for all $h, l \in H$,

$$
\lambda\left(h l_{2}\right) l_{1}=\lambda\left(h_{2} l\right) S\left(h_{1}\right) \text { and } \lambda\left(h l_{1}\right) l_{2}=\lambda\left(h_{1} l\right) S^{-1}\left(h_{2}\right) a^{-1} .
$$

For $\Gamma$ either a nonzero left or right integral for $H$ in $H^{* \text { rat }}$, there are bijective maps from $H$ to $H^{* \text { rat }}$ given by

$$
h \mapsto(h \rightarrow \Gamma) \text { and } h \mapsto(\Gamma<h) .
$$

The first author's research is supported by NSERC.

The second author had support from a PDF at Mount Allison University. He thanks Mount Allison University (Canada) for their warm hospitality. 
Let $\chi$ denote the generalized Frobenius automorphism of $H$ defined in [1], that is, for $\lambda \in \int_{l}^{H^{*}}, \chi$ is the algebra automorphism of $H$ defined by

$$
h \rightarrow \lambda=\lambda<\chi(h) \text {, for all } h \in H .
$$

Then the algebra map $\alpha:=\varepsilon \circ \chi \in H^{*}$ is called the modular element for $H$ in $H^{*}$, terminology justified by the finite dimensional case; see [1, Remark 2.2]. Moreover,

$$
\chi(h)=\alpha\left(h_{2}\right) S^{-2}\left(h_{1}\right) \text { for all } h \in H .
$$

Radford's formula for $S^{4}$ extends to co-Frobenius Hopf algebras as follows.

Theorem 1.1. [1, Theorem 2.8] For $H$ co-Frobenius with modular elements $a \in H$, $\alpha \in H^{*}$, then for all $h \in H$,

$$
S^{4}(h)=a\left(\alpha \rightarrow h<\alpha^{-1}\right) a^{-1}=\alpha^{-1}\left(h_{1}\right) a h_{2} a^{-1} \alpha\left(h_{3}\right) .
$$

Of course, if $H$ is not finite dimensional, then there is no nonzero integral $t \in H$, but nonetheless many of the properties of finite dimensional Hopf algebras which are proved using integrals (such as the $S^{4}$ formula above) carry over to infinite dimensional co-Frobenius Hopf algebras using the integral in $H^{*}$ and the modular elements. Another example, although not directly related to the theme of this paper, is given in the next proposition as further illustration of this point of view.

Proposition 1.2. (cf. [11, Proposition 4]) Let $H$ be co-Frobenius with $\lambda, a, \alpha$ as above. Then the following are equivalent.

(i) $S^{2}$ is co-inner (or equivalently $S^{-2}$ is co-inner).

(ii) There are $\rho, \tau \in(H \otimes H)^{*}$ such that for all $h, l \in H$,

$$
\lambda(l h)=\rho\left(h_{1} \otimes l_{1}\right) \lambda\left(h_{2} l_{2}\right) \tau\left(h_{3} \otimes l_{3}\right) .
$$

Proof. We outline the proof. If $S^{-2}=$ co $-\operatorname{Inn}_{\omega}$, i.e., $S^{-2}(h)=\omega^{-1}\left(h_{1}\right) h_{2} \omega\left(h_{3}\right)$ for some $\omega \in \mathcal{U}\left(H^{*}\right)$, then writing $\lambda(l h)=\lambda(\chi(h) l)$, and using (1.5), it is easy to see that $\lambda(l h)=\rho\left(h_{1} \otimes l_{1}\right) \lambda\left(h_{2} l_{2}\right) \tau\left(h_{3} \otimes l_{3}\right)$ where $\rho=\omega^{-1} \otimes \varepsilon$ and $\tau=(\omega \otimes \varepsilon) *(\alpha \otimes \varepsilon)$.

Conversely, note that $\lambda(l h)=(\lambda<\chi(h))(l)$ and, use (1.2) to obtain

$$
\rho\left(h_{1} \otimes l_{1}\right) \lambda\left(h_{2} l_{2}\right) \tau\left(h_{3} \otimes l_{3}\right)=\rho\left(h_{1} \otimes S\left(h_{2}\right)\right) \lambda\left(h_{3} l\right) \tau\left(h_{5} \otimes S^{-1}\left(h_{4}\right) a^{-1}\right) .
$$

Thus from (1.4) together with (1.3), one obtains that

$$
\chi(h)=\alpha\left(h_{2}\right) S^{-2}\left(h_{1}\right)=\rho\left(h_{1} \otimes S\left(h_{2}\right)\right) h_{3} \tau\left(h_{5} \otimes S^{-1}\left(h_{4}\right) a^{-1}\right)=\rho^{\prime}\left(h_{1}\right) h_{2} \tau^{\prime}\left(h_{3}\right),
$$

where $\rho^{\prime}, \tau^{\prime}$ have the obvious definitions. Thus $S^{-2}(h)=\rho^{\prime}\left(h_{1}\right) h_{2} \tau^{\prime \prime}\left(h_{3}\right)$ where $\tau^{\prime \prime}=\tau^{\prime} * \alpha^{-1}$. Applying $\varepsilon$, we see that $\rho^{\prime} * \tau^{\prime \prime}=\varepsilon$. Now compute $\rho^{\prime} \rightarrow S^{-2}(h)=$ $h \leftarrow \rho^{\prime}$ to see that $\rho^{\prime}=\rho^{\prime} \circ S^{-2}$ and similarly $\tau^{\prime \prime}=\tau^{\prime \prime} \circ S^{-2}$. Then $\tau^{\prime \prime} * \rho^{\prime}(h)=$ $\tau^{\prime \prime} * \rho^{\prime}\left(S^{-2}(h)\right)=\varepsilon(h)$ and so $\rho^{\prime}$ and $\tau^{\prime \prime}$ are inverse in $H^{*}$ and $S^{-2}$, and thus $S^{2}$, is co-inner.

For $(H, R)$ quasitriangular then $S^{2}$ is an inner automorphism induced by $u$ or by $v:=S(u)^{-1}$ defined via $R$. Then $v u=u v$, and $S^{4}=\operatorname{Inn}_{v u}$. As well, (see (2.6) below), $v u \in G(H)$. For $H$ finite dimensional, it was proved independently by Drinfeld [8, Proposition 6.2] and Radford [11, Theorem 2] that $u v=v u=a g_{\alpha}$ where $a \in H$ and $\alpha \in H^{*}$ are the modular elements as above, and $g_{\alpha}$ is a grouplike element defined via $R$ and $\alpha$. In this paper, we extend this formula to not necessarily finite dimensional quasitriangular co-Frobenius Hopf algebras with an analogous result for coquasitriangular co-Frobenius Hopf algebras. Note that this paper takes a new approach and that the proof in the coquasitriangular case cannot be viewed as the formal dual of the proof given in the quasitriangular case. 


\section{2. $S^{4}$ When $(H, R)$ is a CO-Frobenius Quasitriangular Hopf Algebra}

Throughout this section, as well as co-Frobenius, $(H, R)$ will be almost cocommutative or else quasitriangular with modular elements $a \in H$ and $\alpha \in H^{*}$. For example, the tensor product of a finite dimensional quasitriangular Hopf algebra and an infinite dimensional group algebra would satisfy the above conditions.

Recall that, for $H$ a Hopf algebra and $R=R^{1} \otimes R^{2}=r^{1} \otimes r^{2} \in \mathcal{U}(H \otimes H)$, then $(H, R)$ is called almost cocommutative if for all $h \in H$,

$$
\Delta^{\mathrm{cop}}(h)=R \Delta(h) R^{-1} .
$$

Then by results of Drinfeld [8, Proposition 2.2] (who credits Lyubashenko) or Radford [11, Proposition 1], $S^{2}$ is an inner automorphism induced by $u:=S\left(R^{2}\right) R^{1}$ or by $v:=S(u)^{-1}=S\left(U^{1}\right) U^{2}$ where $U:=R^{-1}=U^{1} \otimes U^{2}$, i.e.,

$$
S^{2}(h)=u h u^{-1}=v h v^{-1} \text { for all } h \in H .
$$

Remark 2.1. If $S^{2}=\operatorname{Inn}_{w}$, so that $\lambda \circ S^{2}=a^{-1} \rightarrow \lambda<a=w^{-1} \rightarrow \lambda<w$, by (1.5) we have $\chi\left(w^{-1}\right) w=\chi\left(a^{-1}\right) a=\alpha\left(a^{-1}\right) 1$. If $\varepsilon(w)=1$ then $\alpha\left(w^{-1}\right)=\alpha\left(a^{-1}\right)$.

If, as well, the element $R$ satisfies the following,

$$
\begin{aligned}
& \Delta\left(R^{1}\right) \otimes R^{2}=R^{1} \otimes r^{1} \otimes R^{2} r^{2}, \\
& R^{1} \otimes \Delta\left(R^{2}\right)=R^{1} r^{1} \otimes r^{2} \otimes R^{2}, \\
& \varepsilon\left(R^{1}\right) R^{2}=1, \varepsilon\left(R^{2}\right) R^{1}=1,
\end{aligned}
$$

then $(H, R)$ is called quasitriangular. In this case,

$$
U=S\left(R^{1}\right) \otimes R^{2}=R^{1} \otimes S^{-1}\left(R^{2}\right), \text { so that }(S \otimes S)(R)=R .
$$

Furthermore, for $u=S\left(R^{2}\right) R^{1}$ as defined above,

$$
\Delta(u)=(u \otimes u)\left(R_{21} R\right)^{-1}=\left(R_{21} R\right)^{-1}(u \otimes u), \text { where } R_{21}:=R^{2} \otimes R^{1},
$$

so that $u v=u S(u)^{-1} \in G(H)$.

Remarks 2.2. (i) We note that if a co-inner automorphism $\varphi$ of an almost cocommutative Hopf algebra $(H, R)$ is induced by a grouplike in $H^{0}$ then $\varphi$ is inner. Let $\gamma \in G\left(H^{0}\right)$ with convolution inverse $\gamma^{-1}$ and let $\varphi \in \operatorname{Aut}(H)$ be the co-inner automorphism induced by $\gamma^{-1}$ as in Proposition 1.2 Then

$$
\varphi(h)=\gamma^{-1}\left(h_{1}\right) h_{2} \gamma\left(h_{3}\right)=w h w^{-1}
$$

where $w \in W_{\gamma}=\left\{\gamma^{-1}\left(U^{1}\right) U^{2}, \gamma\left(R^{1}\right) R^{2}, \gamma\left(U^{2}\right) U^{1}, \gamma^{-1}\left(R^{2}\right) R^{1}\right\}$. To see this, note that by (2.1),

$$
\varphi(h)=\left[\gamma^{-1}\left(U^{1} h_{2} R^{1}\right) U^{2} h_{1} R^{2}\right] \gamma\left(h_{3}\right)=\gamma^{-1}\left(U^{1}\right) U^{2} h \gamma^{-1}\left(R^{1}\right) R^{2} .
$$

The rest of the statement is shown by using (2.1) in a similar manner.

(ii) If $(H, R)$ is quasitriangular, the map from $G\left(H^{0}\right)$ to $G(H)$ given by

$$
\eta \mapsto a_{\eta}:=\eta\left(R^{1}\right) R^{2}
$$

is a group homomorphism. Also recall that $(H, \tilde{R})$ is quasitriangular where $\tilde{R}:=$ $R_{21}^{-1}=R^{2} \otimes S\left(R^{1}\right)=S^{-1}\left(R^{2}\right) \otimes R^{1}$, and so there is also a group homomorphism from $G\left(H^{0}\right)$ to $G(H)$ given by

$$
\eta \mapsto b_{\eta}:=\eta\left(S^{-1}\left(R^{2}\right)\right) R^{1}=\eta^{-1}\left(R^{2}\right) R^{1} .
$$

(The proof of this is explicit in [11, Proposition 3].) So in the quasitriangular case, using (2.5) we see that $W_{\gamma}=\left\{a_{\gamma}, b_{\gamma}\right\}$. 
(iii) As in [8, the map from $G\left(H^{0}\right)$ to $G(H)$ defined by $\eta \mapsto a_{\eta} b_{\eta^{-1}}$ is a group homomorphism with image in $G(H) \cap Z(H)$. We will see in the next lemma that under this group homomorphism $\alpha$ maps to 1 . For $H$ finite dimensional, this fact was used by Radford [13] in the proof that a factorizable Hopf algebra is unimodular.

For the remainder of this section, we assume that $(H, R)$ is quasitriangular. Then $S^{4}=\operatorname{Inn}_{u v}=\operatorname{Inn}_{a a_{\alpha}}=\operatorname{Inn}_{a b_{\alpha}}$ and we show that the elements inducing the inner automorphism $S^{4}$ are all equal.

Lemma 2.3. Let $H$ be a co-Frobenius quasitriangular Hopf algebra with modular elements $a \in H$ and $\alpha \in H^{*}$. Then $a_{\alpha}=b_{\alpha}$.

Proof. From Remark 2.1, $\chi\left(u^{-1}\right) u=\alpha\left(a^{-1}\right) 1$ and $\alpha\left(u^{-1}\right)=\alpha\left(a^{-1}\right)$. Now, from (1.4) and (2.6), we have

$$
\chi\left(u^{-1}\right) u=\alpha\left(u_{2}^{-1}\right) S^{-2}\left(u_{1}^{-1}\right) u=\alpha\left(R^{1} r^{2} u^{-1}\right) S^{-2}\left(R^{2} r^{1} u^{-1}\right) u=\alpha\left(a^{-1}\right) a_{\alpha} b_{\alpha^{-1}} .
$$

Since $\alpha\left(a^{-1}\right) \neq 0$ it follows that $a_{\alpha}=b_{\alpha}$, as needed.

Now we prove the main result of this section.

Theorem 2.4. Let $(H, R)$ be a co-Frobenius quasitriangular Hopf algebra with modular elements $a \in H$ and $\alpha \in H^{*}$. Then for $u$ and $v=S(u)^{-1}$ as above,

$$
u v=v u=a b_{\alpha}=a a_{\alpha} .
$$

Proof. By Lemma 2.3 it suffices to show that $u v=a b_{\alpha}$.

Let $h \in H$ and $0 \neq \lambda \in \int_{l}^{H^{*}}$. Then, on one hand, we have

$$
\begin{array}{rll}
\lambda\left(R^{2} h_{2}\right) R^{1} h_{1} & \stackrel{2.3}{=} & \lambda\left(h_{1} R^{2}\right) h_{2} R^{1} \\
= & \lambda\left(h_{1} R_{1}^{2}\right) h_{2} R_{2}^{2} S\left(R_{3}^{2}\right) R^{1} \\
& \stackrel{1.1}{=} & l\left(h R_{1}^{2}\right) a^{-1} S\left(R_{2}^{2}\right) R^{1} \\
& \stackrel{2.3}{=} & \lambda\left(h r^{2}\right) a^{-1} S\left(R^{2}\right) R^{1} r^{1}=\lambda\left(h r^{2}\right) a^{-1} u r^{1} .
\end{array}
$$

On the other hand, we compute

$$
\begin{array}{rll}
\lambda\left(R^{2} h_{2}\right) R^{1} h_{1} & = & \lambda\left(R_{3}^{2} h_{2}\right) R^{1} S\left(R_{1}^{2}\right) R_{2}^{2} h_{1} \\
\left(\lambda \in \int_{l}^{H^{*}}\right) & = & \lambda\left(R_{2}^{2} h\right) R^{1} S\left(R_{1}^{2}\right) \\
& 2.3 & \lambda\left(R^{2} h\right) R^{1} r^{1} S\left(r^{2}\right) \\
& \stackrel{2.5}{=} & \lambda\left(R^{2} h\right) R^{1} S\left(S\left(r^{2}\right) r^{1}\right)=\lambda\left(R^{2} h\right) R^{1} S(u) .
\end{array}
$$

Now, by (1.4) and (1.5) we have $\lambda\left(h h^{\prime}\right)=\alpha\left(h_{2}^{\prime}\right) \lambda\left(S^{-2}\left(h_{1}^{\prime}\right) h\right)$, for all $h, h^{\prime} \in H$ or, equivalently, $\lambda\left(h^{\prime} h\right)=\alpha^{-1}\left(h_{2}^{\prime}\right) \lambda\left(h S^{2}\left(h_{1}^{\prime}\right)\right)$, for all $h, h^{\prime} \in H$, and therefore

$$
\begin{array}{rll}
(\lambda \leftarrow h)\left(r^{2}\right) a^{-1} u r^{1} & =\quad \lambda\left(R^{2} h\right) R^{1} S(u) \\
& =\quad \alpha^{-1}\left(R_{2}^{2}\right) \lambda\left(h S^{2}\left(R_{1}^{2}\right)\right) S\left(u S^{-1}\left(R^{1}\right)\right) \\
= & \alpha^{-1}\left(S^{2}\left(R_{2}^{2}\right)\right) \lambda\left(h S^{2}\left(R_{1}^{2}\right)\right) S(u) S^{2}\left(R^{1}\right) \\
& 2.5 & \alpha^{-1}\left(R_{2}^{2}\right) \lambda\left(h R_{1}^{2}\right) S(u) R^{1} \\
= & \alpha^{-1}\left(R^{2}\right) \lambda\left(h r^{2}\right) S(u) R^{1} r^{1} \\
= & (\lambda \leftarrow h)\left(r^{2}\right) S(u) b_{\alpha} r^{1},
\end{array}
$$

for all $h \in H$. Since $H^{* \text { rat }}$ is dense in $H^{*}$ in the finite topology (for example see [4]), we may choose $h \in H$ such that $r^{1}(\lambda<h)\left(r^{2}\right)=r^{1} \varepsilon\left(r^{2}\right)=1$, and then we 
have that $a^{-1} u=S(u) b_{\alpha}=v^{-1} b_{\alpha}$. Finally, since $v^{-1}$ commutes with grouplikes, we obtain that $u v=a b_{\alpha}$, so the proof is complete.

As in the finite dimensional case, Theorem 2.4 implies the following.

Corollary 2.5. Let $(H, R)$ be a co-Frobenius quasitriangular Hopf algebra.

(i) If $\alpha=\varepsilon$ then $S(u)^{-1} u=v u=a$, so $S(u)^{-1} u$ does not depend on $R$.

(ii) $S(u)=u$ if and only if $a_{\alpha}=a^{-1}$.

Every subHopf algebra of a co-Frobenius Hopf algebra is co-Frobenius [4, 5.3.3]. If $0 \neq \lambda \in \int_{l}^{H^{*}}$ and $\lambda(x) \neq 0$ for some $x \in L$, a subHopf algebra of $H$, then $\int_{l}^{H^{*}}=\int_{l}^{L^{*}}=k \lambda$, and the modular elements are $a_{L}=a_{H}=\lambda\left(x_{1}\right) x_{2}$, and $\alpha_{L}=\alpha_{H}$ restricted to $L$. However in general the spaces of integrals do not coincide (in fact, $\operatorname{Ker}(\lambda)$ is a subcoalgebra of codimension 1 ) and the modular elements differ. If $L$ is the minimal quasitriangular subHopf algebra of $H$ (see [12]), then we have the following.

Corollary 2.6. For $(L, R) \subseteq(H, R)$ minimal quasitriangular, then $a_{L}=a_{H}$ if and only if $\alpha_{L}=\alpha_{H}$ restricted to $L$ if and only if $\chi_{L}=\chi_{H}$ where $\chi$ is the generalized Nakayama automorphism defined in (1.4).

Proof. By Theorem 2.4, $a_{L}=a_{H}$ if and only if $a_{\alpha_{L}}=a_{\alpha_{H}}$. Write $R=\sum_{i=1}^{m} u_{i} \otimes$ $v_{i}$ with the sets of $u_{i}$ and of $v_{i}$ linearly independent (cf. [12]). The minimal quasitriangular Hopf algebra $L$ is generated by the $u_{i}$ and $v_{i}$. Then $a_{\alpha_{L}}=a_{\alpha_{H}}$ if and only if $\alpha_{L}$ and $\alpha_{H}$ agree on the $v_{i}$ and $b_{\alpha_{L}}=b_{\alpha_{H}}$ if and only if $\alpha_{L}$ and $\alpha_{H}$ agree on the $u_{i}$. The last equivalence follows from the definition of the generalized Nakayama automorphism.

Note that in the above corollary it is important that $(L, R)$ be minimal quasitriangular. Let $\operatorname{char}(k)$ be different from 2, and let $H=H_{4}$, Sweedler's 4dimensional Hopf algebra generated by the grouplike $g$ and the $(1, g)$-primitive $x$. It is well known that $(H, R)$ is quasitriangular with $R=c+\xi b$ where $c=$ $\frac{1}{2}(1 \otimes 1+1 \otimes g+g \otimes 1-g \otimes g)$ and $b=\frac{1}{2}(x \otimes x-x \otimes g x+g x \otimes x+g x \otimes g x)$. If $\xi \neq 0$, then $a_{H}=g$; if $\xi=0, a_{H}=1$. If $\xi \neq 0$, then $\alpha_{H}\left(g^{i} x^{j}\right)=\delta_{j, 0}(-1)^{i}$; if $\xi=0$, then $\alpha_{H}=\varepsilon$. Now the semisimple cosemisimple subHopf algebra $L=k[\langle g\rangle]$ has trivial modular elements and so these agree with those of $H$ if $\xi=0$ so that $L$ is minimal quasitriangular, but otherwise they are different.

\section{3. $S^{4}$ When $(H, \sigma)$ is a Co-Frobenius coquasitriangular Hopf algebra}

Recall that for $H$ a Hopf algebra and $\sigma: H \otimes H \rightarrow k$, then $(H, \sigma)$ is called coquasitriangular if for all $h, l, m \in H$, the map $\sigma$ satisfies:

$$
\begin{aligned}
& \sigma(h l, m)=\sigma\left(h, m_{1}\right) \sigma\left(l, m_{2}\right), \\
& \sigma(h, l m)=\sigma\left(h_{1}, m\right) \sigma\left(h_{2}, l\right), \\
& \sigma(h, 1)=\sigma(1, h)=\varepsilon(h), \\
& l_{1} h_{1} \sigma\left(h_{2}, l_{2}\right)=\sigma\left(h_{1}, l_{1}\right) h_{2} l_{2} .
\end{aligned}
$$

These conditions imply that $\sigma$ is convolution invertible with inverse $\sigma \circ(S \otimes \mathrm{Id})=$ $\sigma \circ\left(\operatorname{Id} \otimes S^{-1}\right)$, and so $\sigma=\sigma \circ(S \otimes S)$. If only (3.4) holds and $\sigma$ is invertible, then $(H, \sigma)$ is called almost commutative. 
Let $(H, \sigma)$ be coquasitriangular. Let $u \in H^{*}$ be defined by $u(h):=\sigma\left(h_{2}, S\left(h_{1}\right)\right)$ with convolution inverse $u^{-1}=\sigma\left(S^{2}\left(h_{2}\right), h_{1}\right)$. Similarly, define $v(h):=(u \circ S)(h)=$ $\sigma\left(h_{1}, S\left(h_{2}\right)\right)$ with convolution inverse $v^{-1}(h)=\sigma\left(S^{2}\left(h_{1}\right), h_{2}\right)$. Then by the argument in [7, $S^{2}=\mathrm{co}-\operatorname{Inn}_{u}=\mathrm{co}-\operatorname{Inn}_{v^{-1}}$, that is,

$$
S^{2}(h)=u\left(h_{1}\right) h_{2} u^{-1}\left(h_{3}\right)=v^{-1}\left(h_{1}\right) h_{2} v\left(h_{3}\right) .
$$

In particular, $u$ and $v^{-1}$ commute.

Remark 3.1. Note that if $S^{2}=\mathrm{co}-\operatorname{Inn}_{\omega}$ with $\omega(1)=1$, then $\lambda \circ S^{2}=\omega * \lambda * \omega^{-1}=$ $\omega^{-1}\left(a^{-1}\right) \lambda$. But as in Remark 2.1, $\lambda \circ S^{2}=\alpha\left(a^{-1}\right) \lambda$ and so $\omega^{-1}\left(a^{-1}\right)=\alpha\left(a^{-1}\right)$.

The analogue of Remarks 2.2 in the coquasitriangular setting follow.

Remarks 3.2. (i) If $(H, \sigma)$ is almost commutative, then an inner automorphism induced by a grouplike element is co-inner. For, let $g \in G(H)$ and let $\operatorname{Inn}_{g} \in \operatorname{Aut}(H)$ be the inner automorphism of $H$ induced by $g$. Then it is easy to check using (3.4) that $\operatorname{Inn}_{g}=\mathrm{co}-\operatorname{Inn}_{\omega}$ where $\omega \in \mathcal{W}_{g}=\left\{\sigma(-, g), \sigma^{-1}(g,-), \sigma\left(g^{-1},-\right), \sigma^{-1}\left(-, g^{-1}\right)\right\}$.

(ii) Now let $(H, \sigma)$ be coquasitriangular. For $g \in G(H)$, define grouplike elements $\alpha_{g}$ and $\beta_{g}$ in $H^{0}$ by $\alpha_{g}=\sigma\left(-, g^{-1}\right)$ and $\beta_{g}=\sigma(g,-)$. It is easy to check that $\alpha_{g}$ and $\beta_{g}$ lie in $G\left(H^{0}\right)$ and that the maps from $G(H)$ to $G\left(H^{0}\right), g \mapsto \alpha_{g}$ and $g \mapsto \beta_{g}$ are well defined group homomorphisms. Furthermore, here $\mathcal{W}_{g}=\left\{\alpha_{g}, \beta_{g}\right\}$.

Now we have the main result of this section.

Theorem 3.3. Let $(H, \sigma)$ be a co-Frobenius coquasitriangular Hopf algebra with modular elements $a \in H$ and $\alpha \in H^{*}$ and with $u, v$ as defined above. Then

$$
u^{-1} * v=v * u^{-1}=\alpha * \beta_{a}=\alpha * \alpha_{a} .
$$

Proof. For $h, l \in H$, we compute $e=\lambda\left(h l_{1}\right) \sigma\left(l_{3}, a^{-1} S\left(l_{2}\right)\right)$ in two different ways. First, we compute

$$
e^{\stackrel{\sqrt[3.2]{=}}{=}} \lambda\left(h l_{1}\right) \sigma\left(l_{3}, S\left(l_{2}\right)\right) \sigma\left(l_{4}, a^{-1}\right)=\left((\lambda<h) * u * \alpha_{a}\right)(l) .
$$

Next, we see that

$$
\begin{aligned}
e \stackrel{1.1}{=} & \sigma\left(l_{2}, \lambda\left(h_{1} l_{1}\right) h_{2}\right) \stackrel{3.4}{=} \sigma\left(l_{1}, h_{1}\right) \lambda\left(l_{2} h_{2}\right) \\
& =\sigma\left(l_{1}, \lambda\left(l_{4} h_{2}\right) S\left(l_{2}\right) l_{3} h_{1}\right)=\sigma\left(l_{1}, S\left(l_{2}\right)\right) \lambda\left(l_{3} h\right)=(v *(h \rightarrow \lambda))(l) .
\end{aligned}
$$

But it is easy to prove directly (or see the second equation in (1.2)), that for all $p \in H^{*}$, we have $(\lambda \leftarrow h) * p=\lambda \leftarrow p\left(S^{-1}\left(h_{2}\right) a^{-1}\right) h_{1}$, and therefore

$$
(\lambda<h) * u * \alpha_{a}=\lambda<\left(u * \alpha_{a}\right)\left(S^{-1}\left(h_{2}\right) a^{-1}\right) h_{1} .
$$

Similarly, since $p *(h \rightarrow \lambda)=p\left(S^{-1}\left(h_{1}\right)\right) h_{2} \rightarrow \lambda$, for any $p \in H^{*}$, we get that

$$
v *(h \rightarrow \lambda)=(u \circ S) *(h \rightarrow \lambda)=u\left(h_{1}\right) h_{2} \rightarrow \lambda=\lambda\left\llcorner u\left(h_{1}\right) S^{-2}\left(h_{2}\right) \alpha\left(h_{3}\right) .\right.
$$

Thus $\left(u * \alpha_{a}\right)\left(S^{-1}\left(h_{2}\right) a^{-1}\right) h_{1}=u\left(h_{1}\right) S^{-2}\left(h_{2}\right) \alpha\left(h_{3}\right)$. The fact that $u * \alpha=v * \beta_{a^{-1}}$ will follow by applying $\varepsilon$ to this equality, and using the facts that $v=u \circ S=u \circ S^{-1}$, $a \in G(H)$ and $\alpha_{a} \in G\left(H^{0}\right)$. For all $h \in H$,

$$
\begin{aligned}
(u * \alpha)(h)= & \left(u * \alpha_{a}\right)\left(S^{-1}(a h)\right) \\
= & \alpha_{a^{-1}}\left(a h_{1}\right) v\left(a h_{2}\right)=\sigma\left(a h_{1}, a\right) \sigma\left(a h_{2}, S\left(a h_{3}\right)\right) \\
& =\sigma\left(a h_{1}, S\left(h_{2}\right)\right) \\
\stackrel{3.1}{=} & \sigma\left(a h_{1}, S\left(a h_{2}\right) a\right)=\left(a, S\left(h_{3}\right)\right) \sigma\left(h_{1}, S\left(h_{2}\right)\right)=\left(v * \beta_{a^{-1}}\right)(h),
\end{aligned}
$$

and thus $u^{-1} * v=\alpha * \beta_{a}$. 
Recall that $(H, \tilde{\sigma})$ is also coquasitriangular where $\tilde{\sigma}=\sigma^{-1} \circ$ tw, for tw the twist map. It is easily checked that $\tilde{u}=v^{-1}, \tilde{v}=u^{-1}, \widetilde{\alpha_{a}}=\beta_{a}$, and $\widetilde{\beta_{a}}=\alpha_{a}$. Then $\tilde{u}^{-1} * \tilde{v}=\alpha * \widetilde{\beta_{a}}$ yields $v * u^{-1}=\alpha * \alpha_{a}$, and the proof is complete.

Dual to Lemma 2.3 and Corollary 2.5 we have the following.

Corollary 3.4. Let $(H, \sigma)$ be a co-Frobenius coquasitriangular Hopf algebra and $a \in G(H)$ and $\alpha \in G\left(H^{0}\right)$ as above. Then:

(i) $\alpha_{a}=\beta_{a}$.

(ii) If $a=1$ then $u^{-1} * v=\alpha$, so $u^{-1} *(u \circ S)$ does not depend on $\sigma$.

(iii) $u \circ S=u$ if and only if $\alpha_{a}=\alpha^{-1}$.

Examples of quasitriangular co-Frobenius Hopf algebras include the cosemisimple Hopf algebra $S L_{q}(2)$. See [1, Example 2.12] for the computation of the modular elements; the quasitriangular structure comes from the FRT construction and can be found, for example, in [9]. Another example is the following.

Example 3.5. Let $H$ be the Hopf algebra generated as an algebra by the grouplike element $g$ and the $(1, g)$-primitive element $x$ with $g x=-x g, x^{2}=0$. (See [2, Section 4] for details.) Then $H$ is an infinite dimensional co-Frobenius Hopf algebra with $0 \neq \lambda \in \int_{l}^{H^{*}}$ defined by $\lambda\left(g^{i} x^{j}\right)=\delta_{i,-1} \delta_{j, 1}$. The modular elements are $a=g^{-1}$ and $\alpha$ defined by $\alpha\left(g^{i} x^{j}\right)=\delta_{j, 0}(-1)^{i}$. Define $\sigma: H \otimes H \rightarrow k$ by $\sigma\left(g^{i} x^{j}, g^{t} x^{s}\right)=$ $\delta_{s, 0} \delta_{j, 0}(-1)^{i t}$. It is straightforward to check that $(H, \sigma)$ is coquasitriangular. Here $u=v=u^{-1}=v^{-1}, \beta_{a}\left(g^{i} x^{j}\right)=\delta_{j, 0}(-1)^{-i}=\delta_{j, 0}(-1)^{i}=\alpha_{a}\left(g^{i} x^{j}\right)$.

Of course, if $(H, R)$ is quasitriangular, and $L$ is a Hopf algebra contained in $H^{*}$, then $(L, \sigma)$ is coquasitriangular where $\sigma(\alpha, \beta)=\alpha\left(R^{1}\right) \beta\left(R^{2}\right)$. An interesting approach to the converse is via multiplier Hopf algebras.

The results of Drinfeld discussed in this paper have been extended in [6] to discrete quasitriangular multiplier Hopf algebras as defined in [16]. A discrete multiplier Hopf algebra $A$ is one with a cointegral, so if $A$ has a $1, A$ is a finite dimensional Hopf algebra. For $H$ a co-Frobenius Hopf algebra, then $H^{* \text { rat }}$ is a regular discrete multiplier Hopf algebra with integrals, giving interesting infinite dimensional examples in this context. The basic theory of multiplier Hopf algebras is explained in 15, that of multiplier Hopf algebras with integrals and their modular elements in [5] where the analogue of Radford's formula for $S^{4}$ is proved.

Example 3.6. [6, Section 2] For $H$ as in Example 3.5, let $A=\{\lambda<h \mid h \in H\}=$ $H^{* \text { rat }}$. Then it is shown in [6] that $A$ is a discrete quasitriangular multiplier Hopf algebra with integrals. Let $\omega_{p} \in A$ map $g^{i} x^{j}$ to $\delta_{i, p} \delta_{j, 0}$. The modular element in $M(A)$ is $a=\sum_{p \in \mathbb{Z}}(-1)^{p} \omega_{p}$; the modular element in $M(\hat{A})$ is $g^{-1}$. (Recall that by duality for multiplier Hopf algebras the dual multiplier Hopf algebra $\hat{A} \cong H$.) The multiplier $R=R^{-1}=\sum_{p \in \mathbb{Z}} a^{p} \otimes \omega_{p} \in M(A \otimes A)$ makes $(A, R)$ quasitriangular. The dual to $R$ is the coquasitriangular structure from Example 3.5 .

For $(H, \sigma)$ coquasitriangular and co-Frobenius, consider the multiplier Hopf algebra $A=H^{* \text { rat }}=\lambda \leftarrow H$. Let $R=\sigma \in(H \otimes H)^{*}$. Since $\sigma *(\lambda \leftarrow h \otimes \lambda \leftarrow l)=$ $\sigma\left(h_{1}, l_{1}\right)\left(\lambda \leftarrow h_{2} \otimes \lambda \leftarrow l_{2}\right)$ and $(\lambda \leftarrow h \otimes \lambda<l) * \sigma=\sigma\left(a h_{2}, a l_{2}\right)\left(\lambda \leftarrow h_{1} \otimes \lambda \leftarrow l_{1}\right)$ by (1.2), then $\sigma \in M(A \otimes A):=\left\{m \in(H \otimes H)^{*} \mid m(A \otimes A),(A \otimes A) m \subseteq A \otimes A\right\}$. A similar computation shows that $(\lambda\llcorner h \otimes \varepsilon) * \sigma \in A \otimes M(A)$ with similar variations on the left and right. The defining properties for quasitriangularity [6, 2.3] then appear to follow from the properties of $\sigma$. 


\section{REFERENCES}

[1] M. Beattie, D. Bulacu and B. Torrecillas, Radford's $S^{4}$ formula for co-Frobenius Hopf algebras, J. Algebra 307 (2007), 330-342.

[2] M. Beattie, S. Dăscălescu, L. Grünenfelder and C. Năstăsescu, Finiteness conditions, coFrobenius Hopf algebras, and quantum groups, J. Algebra 200 (1998), 312-333.

[3] M. Beattie, S. Dăscălescu and Ş. Raianu, Galois Extensions for co-Frobenius Hopf algebras, J. Algebra 198 (1997), 164-183.

[4] S. Dăscălescu, C. Năstăsescu and Ş. Raianu, "Hopf Algebras: An Introduction", in: Monographs Textbooks in Pure Appl. Math., Vol. 235, Dekker, New York, 2001.

[5] L. Delvaux, A. van Daele and S. Wang, A note on Radford's formula, arXiv:math.RA/060809

[6] L. Delvaux, A. Van Daele and S. Wang, Quasitriangular (G-cograded) multiplier Hopf algebras, J. Algebra 289 (2005), 484-514.

[7] Y. Doi, Braided bialgebras and quadratic bialgebras, Comm. Algebra 21(5) (1993), 17311749.

[8] V. G. Drinfeld, On almost cocommutative Hopf algebras, Leningrad Math. J. 1 (1990), 321342.

[9] C. Kassel, "Quantum Groups", Graduate Texts in Mathematics 155, Springer Verlag, Berlin, 1995.

[10] S. Montgomery, "Hopf algebras and their actions on rings", American Mathematical Society, Providence, 1993.

[11] D. E. Radford, On the antipode of a quasitriangular Hopf algebra, J. Algebra 151 (1992), $1-11$.

[12] D. E. Radford, Minimal quasitriangular Hopf algebras, J. Algebra 157 (1993), 285-315.

[13] D. E. Radford, On Kauffman's knot invariants arising from finite-dimensional Hopf algebras, Advances in Hopf algebras (Chicago, IL, 1992), 205-266, Lecture Notes in Pure and Appl. Math. 158, Dekker, New York, 1994.

[14] M. E. Sweedler, "Hopf algebras", Benjamin, New York, 1969.

[15] A. Van Daele, Multiplier Hopf algebras, Trans. Amer. Math. Soc. 342 (1994), 917-932.

[16] Yinhuo Zhang, The quantum double of a coFrobenius Hopf algebra, Communications in Algebra, 27 (1999), 1413-1427.

Department of Mathematics and Computer Science, Mount Allison University, SaCKVille, NB E4L 1E6, CANAdA

E-mail address: mbeattie@mta.ca

Faculty of Mathematics and Informatics, University of Bucharest, Str. Academiei 14, RO-010014 Bucharest 1, ROMANia

E-mail address: dbulacu@al.math.unibuc.ro 